\title{
Simulations of XUV Disks with a Star Formation Density Threshold
}

\section{Citation}

Bush, Stephanie J., T. J. Cox, Lars Hernquist, David Thilker, and Joshua D. Younger. 2008. "Simulations of XUV Disks with a Star Formation Density Threshold." The Astrophysical Journal 683 (1): L13-16. https://doi.org/10.1086/591523.

\section{Permanent link}

http://nrs.harvard.edu/urn-3:HUL.InstRepos:41381762

\section{Terms of Use}

This article was downloaded from Harvard University's DASH repository, and is made available under the terms and conditions applicable to Open Access Policy Articles, as set forth at http:// nrs.harvard.edu/urn-3:HUL.InstRepos:dash.current.terms-of-use\#OAP

\section{Share Your Story}

The Harvard community has made this article openly available.

Please share how this access benefits you. Submit a story.

Accessibility 
ACCEPTEd For PUblicATion in ApJL

Preprint typeset using $\mathrm{LAT}_{\mathrm{E}} \mathrm{X}$ style emulateapj v. 08/22/09

\title{
SIMULATIONS OF XUV DISKS WITH A STAR FORMATION DENSITY THRESHOLD
}

\author{
Stephanie J. Bush ${ }^{1}$, T.J. Cox ${ }^{1,2}$, Lars Hernquist ${ }^{1}$, David Thilker ${ }^{3}$, Joshua D. Younger ${ }^{1}$ \\ Accepted for publication in ApJL
}

\begin{abstract}
The outer regions of disk galaxies show a drop-off in optical and $\mathrm{H} \alpha$ emission, suggesting a star formation threshold radius, assumed to owe to a critical surface density below which star formation does not take place. Signs of filamentary star formation beyond this threshold radius have been observed in individual galaxies in the $\mathrm{H} \alpha$ and recent GALEX surveys have discovered that $30 \%$ of disk galaxies show UV emission out to 2-3 times the optical radius of the galaxy. We run smooth particle hydrodynamics simulations of disk galaxies with constant density extended gas disks to test whether over-densities owing to spiral structure in the outer disk can reproduce the observed star formation. We indeed find that spiral density waves from the inner disk propagate into the outer gas disk and raise local gas regions above the star formation density threshold, yielding features similar to those observed. Because the amount of star formation is low, we expect to see little optical emission in outer disks, as observed. Our results indicate that XUV disks can be simulated simply by adding an extended gas disk with a surface density near the threshold density to an isolated galaxy and evolving it with fiducial star formation parameters.
\end{abstract}

Subject headings: galaxies: spiral, galaxies: structure, galaxies: evolution, ultraviolet: galaxies

\section{INTRODUCTION}

The most basic description of galactic-scale star formation is the Schmidt relation, which assumes that the star-formation rate (SFR) is a power law function of the gas density (Schmidt 1959). Kennicutt (1998) showed that this relation accurately describes star formation in galaxies over a range of $10^{5}$ in gas surface density and $10^{6}$ in SFR per unit area. However, beyond a critical galactocentric radius, $\mathrm{SFR}$ determined from $\mathrm{H} \alpha$ emission are truncated relative to the expectation from the Kennicutt-Schmidt relation despite the availability of large reserves of low density cold gas (Kennicutt 1989; Martin \& Kennicutt 2001; Wong \& Blitz 2002). The lack of star formation at large radii has primarily been attributed to dynamical disk stability, i.e.,

$$
Q(r)=\frac{\Sigma_{c}(r)}{\Sigma_{\text {gas }}(r)}=\frac{\kappa(r) c_{s}}{\pi G \Sigma_{\text {gas }}(r)} \gtrsim 1,
$$

where $\kappa$ is the epicyclic frequency at a given radius, $\Sigma_{c}(r)$ is the critical surface density and $c_{s}$ is sound speed of the gas, proportional the velocity dispersion of the gas ( $\sigma=c_{s} \gamma^{-1 / 2}$, where $\gamma$ is the ratio of specific heats) (Toomre 1964; Spitzer 1968; Quirk 1972). The effective velocity dispersion of gas in spirals is roughly constant across the disk (e.g., van der Kruit \& Shostak 1984; Andersen et al. 2006) leaving $\kappa$ as the only radially dependent quantity in the critical surface density. If velocity curves of spiral galaxies are approximately flat, $\kappa$, and therefore $\Sigma_{c}$, fall with $r$, implying that at some radius the disk becomes gravitationally stable, which may inhibit star formation. In support of this

\footnotetext{
Electronic address: sbush@cfa.harvard.edu

${ }^{1}$ Harvard-Smithsonian Center for Astrophysics, 60 Garden St, Cambridge, MA 02143 USA

2 W.M. Keck Postdoctoral Fellow at the Harvard-Smithsonian Center for Astrophysics

3 Center for Astrophysical Sciences, The Johns Hopkins University, 3400 North Charles ST, Baltimore, MD 21218
}

notion, several studies have shown correlations between the radius at which star formation ceases and the radius at which $Q \approx 2$ (Kennicutt 1989; Martin \& Kennicutt 2001; Wong \& Blitz 2002, see Schaye (2004) for a comparison of these results). More sophisticated models including the formation of cold molecular gas have also been successful at reproducing observed SFR truncations (Elmegreen \& Parravano 1994; Schave 2004).

Observations indicate the presence of star formation beyond the threshold. Faint, isolated H II regions distributed in spiral structures out to two optical radii have been found in face on galaxies (Ferguson et al. 1998) and in cold disks in edge-on galaxies (Christlein \& Zaritsky 2008). Similar star formation has been seen in the Milky Way (Brand et al. 2001) and dwarf galaxies with gas densities below the threshold (van Zee et al. 1997). A systematic survey by the Galaxy Evolution Explorer (GALEX: Martin et al. (2005)) recently discovered that $30 \%$ of disk galaxies show extended UV emission beyond the optical radius, and presumed star formation threshold, of the galaxy (Thilker et al. 2007; Gil de Paz et al. 2005; Thilker et al. 2005; Zaritsky \& Christlein 2007). In $2 / 3$ of these cases, this emission is structured, lying in spiral or filamentary patterns (Type I XUV disks). In the other $1 / 3$, the emission takes the form of a large zone in the outer regions of the galaxy with an enhanced $\mathrm{UV} /$ optical ratio relative to the inner disk (Type II XUV disk) (Thilker et al. 2007, hereafter T07).

To reconcile outer disk star formation with disk stability, it has been proposed that local densities above $\Sigma_{c}(r)$ in the outer disk allow star formation beyond the truncation radii (Kennicutt 1989; Martin \& Kennicutt 2001; Schave 2004; Elmegreen \& Hunter 2006; Gil de Paz et al. 2007). The low SFR implies a low number of $\mathrm{O}$ stars producing $\mathrm{H} \alpha$ emission at a given time, or none at all, explaining why this star formation was undetected in the $\mathrm{H} \alpha$ in some galaxies (Gil de Paz et al. 2007). Most of these disks have 
large amounts of HI in their outer regions and UV and $\mathrm{H} \alpha$ complexes are often coincident with local H I over-densities (Ferguson et al. 1998, T07) supporting this picture. Analysis of the star formation profiles of these XUV disk galaxies indicates that the azimuthally averaged Kennicutt-Schmidt relation could hold beyond the dynamical threshold radius (Boissier et al. 2007, Hereafter B07). However, studies of star forming clumps in the XUV disk of M 83 indicate that traditional star formation laws, including the threshold radius, hold locally (Dong et al. 2008).

Theoretically, Elmegreen \& Hunter (2006) have shown that XUV disks may not be inconsistent with a star formation density threshold. In their analytical model, gas clumping triggered by spiral density waves, radial variations in the interstellar medium (ISM) turbulence, and gas phase transitions lead to local regions of star formation in the extended gas disk. In this paper, we investigate XUV disks using smooth particle hydrodynamic (SPH) simulations of an isolated disk galaxy with an extended gas disk and a simple, commonly employed, star formation prescription based on the Kennicutt-Schmidt relation that includes a threshold density for star formation. We demonstrate that, even with these fiducial star formation laws, spiral structure propagates from the inner disk to the extended HI disk and produces local regions of enhanced of gas density which trigger star formation similar to that seen in observed Type I XUV disks.

\section{METHOD}

We used the SPH code GADGET2 (Springel 2005) to run simulations of galaxies with extended gas disks. We incorporate a sub-resolution multi-phase model of the ISM according to Springel \& Hernquist (2003), which includes radiative cooling. The parameters of the multiphase model are $t_{\star}=6.5 \mathrm{Gyr}, A_{0}=3000$ and $T_{S N}=$ $3 \times 10^{8}$. The equation of state is determined by these parameters. No additional star formation feedback is employed. Star formation from the cold phase (total cold gas, our simulation does not treat atomic and molecular gas separately) follows a Schmidt volume density law $\rho_{S F R} \propto \rho_{\text {gas }}^{N}$ with $N=1.5$ that is normalized to give approximately the same SFR as the Milky Way. A local star formation volume density cutoff of $0.004 \mathrm{M}_{\odot} / \mathrm{pc}^{3}$ is included to give a surface density threshold of $\sim 5-10$ $\mathrm{M}_{\odot} / \mathrm{pc}^{2}$, consistent with observations (Kennicutt 1989; Martin \& Kennicutt 2001).

The progenitor galaxy models were constructed following Springel et al. (2005). The galaxy is roughly analogous to the Milky Way with a total mass of $M_{200} \sim 10^{12}$ $\mathrm{M}_{\odot}$ composed of a Hernquist (1990) dark matter halo with $c=9$, a stellar disk scale length of $3.7 \mathrm{kpc}$ (we use $h=0.72$ throughout this paper), an exponential gas disk of the same scale length. However, we add an approximately constant density gas disk that extends to $45 \mathrm{kpc}$ and has a surface density of $\sim 3.5 \mathrm{M}_{\odot} / \mathrm{pc}^{2}$. The baryonic mass fraction is 0.04 . Forty percent of the baryons are in the stellar disk, $20 \%$ are in the exponential gas disk and $40 \%$ are in the constant density gas disk. The gas profile of the galaxy at four times in the simulation is shown in Figure1. The galaxy has no bulge and a $0.01 \%$ $M_{200}$ mass black hole in its center. The black hole is represented by a sink particle that can accrete mass at the

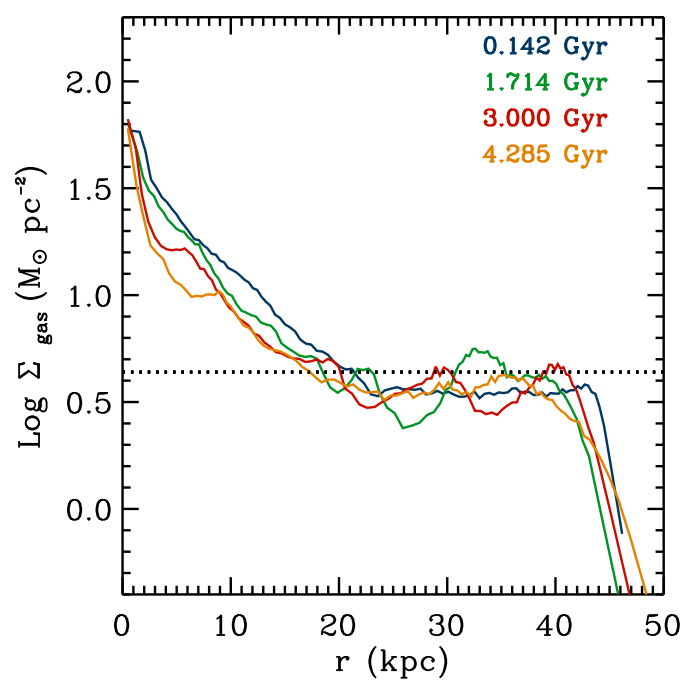

FIG. 1. - Gas density profile of the simulated galaxy at the four different times shown in Figure 2 Notice how in the first simulation snapshot (0.142 Gyr, blue) the extended gas disk has a flat profile, while as the simulation goes on and spiral structure develops, the outer disk shows peaks that lie above the star formation threshold density. From the simulation, the volume density threshold appears to correspond to a surface density threshold of approximately $\sim 4.5$ $\mathrm{M}_{\odot} / \mathrm{pc}^{2}$ which is shown by the dotted line.

Bondi rate (Springel et al.|2005), but as we are interested in the outer disk its presence does not affect our results. The models have $N_{\text {halo }}=1.2 \times 10^{5}, N_{\text {stars }}=8 \times 10^{4}$ and $N_{\text {gas }}=1.6 \times 10^{5}$ particles in each component. The GADGET softening length of the baryonic particles and halo particles are $0.14 \mathrm{kpc}$ and $0.28 \mathrm{kpc}$, respectively.

\section{RESULTS}

The time evolution of our simulated galaxy is shown in Figure 2. The top row shows the stellar surface density, the middle row shows gas surface density, and the bottom row shows the instantaneous SFR surface density, a rough indicator of what is observed in the UV. Since the instantaneous SFR is shown instead of SFR over the last 5-200 Myr, this is a lower limit on the UV emission.

Figure 2 demonstrates that spiral structure from the inner disk propagates to the edge of the extended gas disk. In the outer disk, peaks of the spiral waves that are above the density threshold form stars and the troughs that are below the density threshold do not. This gives filamentary star formation in the outer disk, as seen in Type I XUV disks (T07). Since this is an isolated disk, it shows very regular spiral structure, including ring features seen in the second panel of Figure 2. In reality, peaks of spiral structure in disks will be influenced by interactions with low mass satellites and halo substructure, in addition to the clumpiness of the ISM. The star formation in this model is not sufficient to create a high surface brightness component of stars in the optical, even after 4 Gyr. However, very low surface brightness populations of stars accompany the XUV features. Low surface brightness populations of stars are known to exist in outer parts of disks accompanying UV sources (Zaritsky \& Christlein 2007; Gil de Paz et al. 2007).

\section{DISCUSSION}

4.1. Radial profiles 


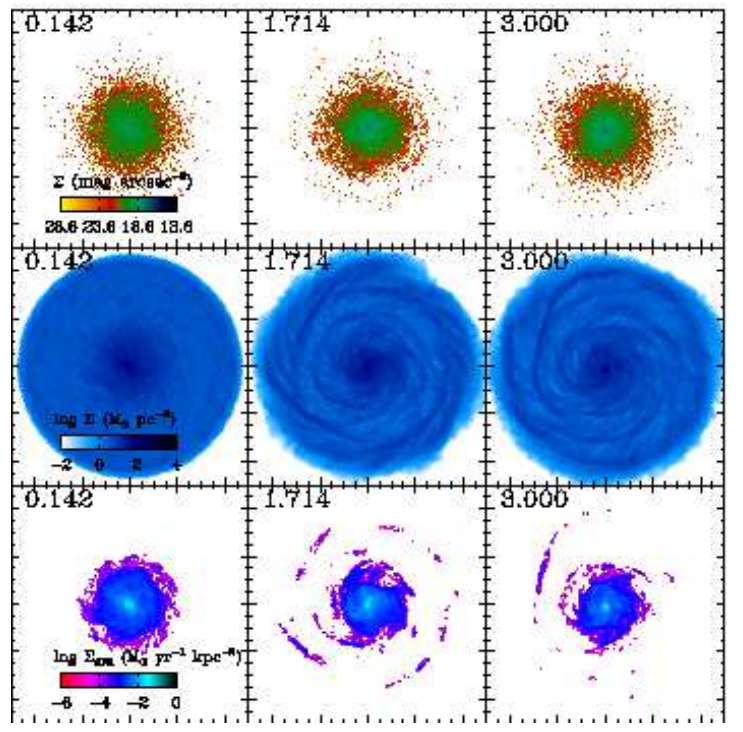

FIG. 2.- Three snapshots from our simulation showing the surface density of stellar particles (top), gas particles (middle) and SFR (proportional to UV luminosity, bottom). Scales for each are shown in the left panel, the color bar for the stellar particle panels is converted to projected $\mathrm{K}$-band surface brightness in units of mag $\operatorname{arcsec}^{-2}$, assuming a constant mass to light ratio of $2 \mathrm{M} \odot / \mathrm{L} \odot$. If averaged radially the outer regions have a surface brightness of $\sim$ $25 \mathrm{mag} \operatorname{arcsec}^{-2}$. SFR is proportional to UV luminosity, so the SFR density range corresponds to a range in UV luminosity range of $7 \times 10^{21}$ to $7 \times 10^{29} \mathrm{ergs} \mathrm{s}^{-1} \mathrm{~Hz}^{-1} \mathrm{kpc}^{-2}$. The panels are 100 $\mathrm{kpc}$ wide. These images illustrate that fiducial star formation laws create XUV morphology; notice how the star formation follows the peaks in the gas density distribution. The optical emission in the outer regions of the galaxy is near or below the surface brightness detection limit for most instruments. However, SFRs seen in the bottom images are detectable, and are similar to those seen in XUV disks (T07, B07). If this simulation were observed, spiral structure in the outer disk would be apparent in UV emission without accompanying K-band emission, as in Type 1 XUV disks.

In the example presented here, we chose a constant density radial profile for the extended gas disk. We ran an additional simulation with only an exponential gas disk of the same scale length as the stellar disk and found that while star formation took place near or slightly beyond the optical radius, the average density of gas at several optical radii is too low to be locally above the star formation threshold, so stars did not form there. The choice of a constant density extended disk was motivated by examples of Type I XUV disks that have fairly constant density extended HI disks (e.g. NGC 4625, NGC 5055, M83; Bush \& Wilcots 2004; Thilker et al. 2005; Bosma 1981). Our simulation is not intended to reproduce all XUV galaxies, but to illustrate that outer disk star formation of the observed morphology is consistent with fiducial star formation laws.

The gas disk we have used for this galaxy model is relatively extended ( $\sim 45 \mathrm{kpc}$ radius), to illustrate that the size of the disk is not an impediment to spiral structure propagating to its edge. The important quantity for comparing to observations is the ratio of the sizes of the optical and gas disks. Assuming that about 2.5 scale lengths of the galaxy would be easily imaged in the optical, the ratio of the gas to optical disk radii is about 5 for this simulation, which is realistic when compared to XUV disks, for example, M 83 (T07).

\subsection{Longevity}

We expect XUV behavior to persist as long as there is spiral structure in the outer disk and the peaks in the density distribution exceed the threshold. Some XUV emission persists throughout the $\sim 4$ Gyr duration of our simulation. The gas density in the outer regions is depleted by less than $1 \%$ during the simulation and therefore XUV behavior will continue for the lifetime of the galaxy, as long as this gas is not removed by another process (such as ram pressure stripping or tidal interactions) and spiral perturbations continue. Although XUV emission is long-lived, its appearance is time-dependent because it follows the spiral perturbations. For example, owing to strong spiral behavior at 1.714 Gyr, as shown in Figure 2 and Figure 1, we see rings of UV emission in the outer disk. At 3.857 Gyr, there is weak spiral structure in the outer disk, leading to only a few spots of UV emission.

In our simulation, the development of spiral structure is seeded by numerical noise arising from the discretized dark matter halo (Hernquist 1993). However, the simulation of XUV disks does not require a particular mechanism for exciting spiral structure. If there is spiral structure in inner disks, as observed, our simulation shows that it easily propagates to the edge of the outer disk and that UV emission tracing that spiral structure can occur.

\subsection{The Kennicutt-Schmidt Relation}

B07 average the gas density and SFR density in elliptical annuli of 43 nearby galaxies ( 14 of which are XUV disks) to determine whether the Kennicutt-Schmidt relation holds when including UV emission. They find that these galaxies fall on the Kennicutt-Schmidt relation and extend to surface densities as low as $0.2 \mathrm{M}_{\odot}$ pc ${ }^{-2}$, but with considerable scatter. Because our simulation has a constant density outer gas density profile at $3.5 \mathrm{M}_{\odot}$ $\mathrm{pc}^{-2}$, moderated only by spiral density waves, we do not probe star formation in such low surface density gas. However, in Figure 3 we present a similar analysis of our simulation, binning one snapshot in two bin widths, 2.8 $\mathrm{kpc}$ and $8.3 \mathrm{kpc}$.

It should be noted that the simulated star formation, shown in Figure 3 is normalized slightly below the empirical Kennicutt-Schmidt law (dotted line) so that the total SFR is approximately that of the Milky Way; however, this will only affect the vertical normalization and not the shape within this plot. In any case, with either the binning, our simulation shows evidence of a cutoff, but the behavior can be surprisingly varied. When binned finely, bins that lie between spiral density waves and therefore below the density threshold, fall off the KennicuttSchmidt relation, so bins near the threshold density show a wide variation in SFR. However, in coarser bins, this effect is smoothed as high and low density regions are included in the same annulus. Depending on the morphology of the spiral density perturbation and the binning chosen, the variation in SFR near the threshold density can be smoothed to the point where these features appear to be scatter around a Kennicutt-Schmidt relation. We conclude that data in a Kennicutt-Schmidt plot can be influenced by such factors as the binning in addition to the underlying star formation process and need to be interpreted carefully. The Kennicutt-Schmidt plot behavior is likely to depend sensitively on the gas dis- 


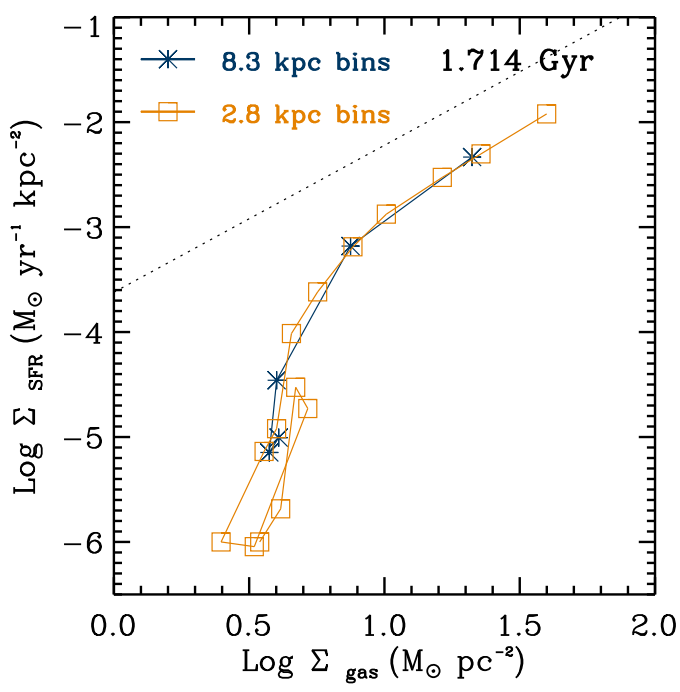

FIG. 3.- Kennicutt-Schmidt relation plotted for one snapshot of our simulation. The gas density and SFR density are averaged in rings at two different bin widths out to $42 \mathrm{kpc}$. Note that annuli with a value of $10^{-6} \mathrm{M}_{\odot} / \mathrm{pc}^{-2}$ are upper limits on the star formation rate.

tribution and more simulations are needed to determine whether the B07 results can be reproduced with fiducial star formation laws.

\section{CONCLUSIONS}

We show that features similar to those seen in Type I XUV disks can be produced with fiducial star formation prescriptions, including a star formation threshold density, if there is gas present at large radii near the threshold density. This is a clear illustration of the fact that a star formation threshold density does not imply the existence of a star formation threshold radius beyond which star formation ceases. As proposed in earlier studies and shown by Elmegreen \& Hunter (2006), local over-densities, caused by spiral density waves in our simulation, allow star formation in gas that, when circularly averaged, is below or near the classical star formation threshold density.

It is possible that star formation will differ between inner and outer disks. This could arise owing to different properties of the ISM in the inner and outer regions of disks, from, for example, a different ionized fraction owing to various levels of self-shielding against an ionizing UV background. Currently, our simulations do not have the resolution to probe the detailed physics of star formation. Here, we simply show that any simulation whose star formation prescription follows a Kennicutt-Schmidt relation with a star formation threshold density and has a high enough gas density for local over-densities to exceed the star formation threshold density in the outer disk will exhibit UV emission in the outer disk.

Understanding the mechanism for generating XUV disks has promising implications for probing galaxy structure in the outer parts of galaxies. If the distribution of H I can be determined based on the observed UV emission, this can be used to infer the extended H I properties of disks. Outer disk H I and its star formation history could also constrain recent gas accretion in galaxies and the formation theory of disks. For these reasons, the generation of XUV disks warrants further exploration. Simulations having different extended gas profiles (e.g. exponential, $1 / R$ ) at various gas densities are needed to test whether we can create the range of properties seen in XUV disks, including Type II XUV disks. Upcoming extragalactic H I surveys such as THINGs (Walter et al. 2005) and ALFALFA (Giovanelli et al. 2005) will provide better statistics on the types of $\mathrm{HI}$ profiles seen on disk galaxies and how they correlate with XUV disk behavior. The role of interactions, particularly flybys, in exciting spiral structure and affecting the morphology of XUV disks also needs to be explored. Finally, the application of a radiative transfer code to our SPH results to compare colors of these clumps to samples such as T07 and Zaritsky \& Christlein (2007) would provide interesting constraints on star formation such as the initial mass function in outer disks.

We appreciate the helpful comments of the referee, Joop Schaye. We thank Phil Hopkins, Dusan Keres, Zhong Wang and Jacqueline van Gorkom for useful conversations in the preparing this work. Support for TJC was provided by the W.M. Keck Foundation.

\section{REFERENCES}

Andersen, D. R. et al. 2006, ApJS, 166, 505

Boissier, S. et al. 2007, ApJS, 173, 524

Bosma, A. 1981, AJ, 86, 1791

Brand, J., Wouterloot, J. G. A., Rudolph, A. L., \& de Geus, E. J. 2001, A\&A, 377, 644

Bush, S. J. \& Wilcots, E. M. 2004, AJ, 128, 2789

Christlein, D. \& Zaritsky, D. 2008, ArXiv e-prints, 803

Dong, H., Calzetti, D., Regan, M., Thilker, D., Bianchi, L., Meurer, G. R., \& Walter, F. 2008, ArXiv e-prints, 804

Elmegreen, B. G. \& Hunter, D. A. 2006, ApJ, 636, 712

Elmegreen, B. G. \& Parravano, A. 1994, ApJ, 435, L121+

Ferguson, A. M. N., Wyse, R. F. G., Gallagher, J. S., \& Hunter,

D. A. 1998, ApJ, 506, L19

Gil de Paz, A. et al. 2005, ApJ, 627, L29

-. 2007, ApJ, 661, 115

Giovanelli, R. et al. 2005, AJ, 130, 2598

Hernquist, L. 1990, ApJ, 356, 359

-. 1993, ApJS, 86, 389

Kennicutt, Jr., R. C. 1989, ApJ, 344, 685

—. 1998, ApJ, 498, 541
Martin, C. L. \& Kennicutt, Jr., R. C. 2001, ApJ, 555, 301

Martin, D. C. et al. 2005, ApJ, 619, L1

Quirk, W. J. 1972, ApJ, 176, L9+

Schaye, J. 2004, ApJ, 609, 667

Schmidt, M. 1959, ApJ, 129, 243

Spitzer, Jr., L. 1968, (New York, NY (USA): John Wiley \& Sons), No. 28 , XIII + 262 p., 28

Springel, V. 2005, MNRAS, 364, 1105

Springel, V., Di Matteo, T., \& Hernquist, L. 2005, MNRAS, 361, 776

Springel, V. \& Hernquist, L. 2003, MNRAS, 339, 289

Thilker, D. A. et al. 2005, ApJ, 619, L79

-. 2007, ApJS, 173, 538

Toomre, A. 1964, ApJ, 139, 1217

van der Kruit, P. C. \& Shostak, G. S. 1984, A\&A, 134, 258

van Zee, L., Haynes, M. P., Salzer, J. J., \& Broeils, A. H. 1997, AJ, 113, 1618 
Walter, F., Brinks, E., de Blok, W. J. G., Thornley, M. D., \& Kennicutt, R. C. 2005, in Astronomical Society of the Pacific Conference Series, Vol. 331, Extra-Planar Gas, ed. R. Braun, $269-+$
Wong, T. \& Blitz, L. 2002, ApJ, 569, 157

Zaritsky, D. \& Christlein, D. 2007, AJ, 134, 135 